\title{
Pilot-Aided Channel Estimation on SC-PAM Based Visible Light Communications
}

\author{
Omer Narmanlioglu ${ }^{1}$, Bugra Turan ${ }^{2}$, Refik Caglar Kizilirmak ${ }^{3}$, Sinem Coleri Ergen ${ }^{2}$, and Murat Uysal ${ }^{1}$ \\ ${ }^{1}$ Department of Electrical and Electronics Engineering, Ozyegin University, Istanbul, Turkey \\ E-mail: omer.narmanlioglu@ozu.edu.tr, murat.uysal@ozyegin.edu.tr \\ ${ }^{2}$ Department of Electrical and Electronics Engineering, Koc University, Istanbul, Turkey \\ E-mail: bturan14@ku.edu.tr, sergen@ku.edu.tr \\ ${ }^{3}$ Department of Electrical and Electronics Engineering, Nazarbayev University, Astana, Kazakhstan \\ E-mail: refik.kizilirmak@nu.edu.kz
}

\begin{abstract}
Estimation of the time-varying optical wireless channel response is crucial in order to decode received signals coherently. In this work, we investigate symbol-error-rate and mean absolute error performance of different interpolation techniques including linear, nearest, spline, and piece-wise cubic Hermite interpolating polynomial (pchip), which are used in pilot-aided channel estimation process for visible light communication. The performance of interpolators is evaluated in realistic time-varying channel model, generated on Zemax and compared with each other under the consideration of different modulation orders, different pilot symbol periods, and different user equipment (UE) speeds through Monte Carlo simulations. The results reveal that spline and pchip techniques are more robust to low pilot symbol transmission rate and fast time-varying channel conditions as a consequence of high UE speeds. However, low complex linear interpolation technique can be chosen for highly rated pilot signal transmission cases or when optical channel varies slowly over the time.
\end{abstract}

Index Terms-Channel Estimation, Interpolation, Visible Light Communication.

\section{INTRODUCTION}

Visible light communication (VLC) is based on the dual usage of light emitting diodes (LEDs) for both illumination and communication purposes. Since LEDs can be modulated at high speeds that are not noticeable to human eye, they can be used as wireless access points [1]. VLC has been receiving attention as a short-range broadband wireless communication technology. This is likely to further grow along with the recent standardization efforts [2].

Fast time-varying optical wireless channel responses are known to lead error rate increments and error floors, resulting with system performance degradation. Hence, mitigating these degradation is key for reliable transmissions. In order to overcome fast-time varying effects of the channel, dynamic estimation of the optical wireless channel between LEDs and photodetectors (PDs) on the user equipment (UE) is necessary. Dynamic channel estimation aims to equalize the received signal effectively, before demodulation process. The channel

The work of M. Uysal was supported by the Turkish Scientific and Research Council (TUBITAK) under Grant 215E311. Sinem Coleri Ergen acknowledges the financial support by the Turkish Academy of Sciences (TUBA) within the Young Scientist Award Program (GEBIP) and METU-Prof. Dr. Mustafa Parlar Foundation Research Encouragement Award. estimation can be performed by inserting pilot signals to the transmitted frame structure with a certain period that is known by the receiver side. Therefore, receiver first estimates the channel response with the certain period, then interpolates time-varying channel response using a specific interpolation technique. Common interpolation techniques such as linear, nearest, spline interpolation, or piece-wise cubic Hermite interpolating polynomial (pchip) have different implementation and computational complexities and offer different accuracy with respect to the interpolated data type.

Various optical wireless channel estimation schemes in the context of VLC are investigated in the literature [3][12]. Authors in [3] proposed an adaptive equalization system for on-off keying (OOK) modulated multiple input multiple output (MIMO) VLC system. In [4]-[6], authors analyzed the performance of Least Square (LS) and minimum mean square error (MMSE) channel estimation techniques over direct current biased optical OFDM (DCO-OFDM) based single-input single-output (SISO) VLC scheme. In [7], authors proposed a discrete Fourier transform (DFT) based post-processing channel estimation to enhance the performance of conventional LS estimator. Similarly, [8] proposed an adaptive channel estimation algorithm for asymmetrically-clipped optical OFDM (ACO-OFDM) based VLC. Moreover, [9] investigate the impact of different one-dimensional interpolation technique on pilot-aided channel estimation over ACO-OFDM based SISO VLC. [10] proposed sparse Bayesian relevance vector machine regression based channel estimation for orthogonal frequency-division multiplexing (OFDM) based VLC. In [11] and [12], a statistical Bayesian MMSE channel estimator is presented for SISO DCO-OFDM system. However, none of the above works consider realistic time-varying response of optical VLC channel and relatively they cannot perform any estimation process over time-varying channels. In this work, we investigate the symbol-error-rate (SER) and mean absolute error (MAE) performance of different interpolation techniques with respect to varying modulation orders, pilot signal periods, and UE speeds over the realistic time-varying channel model that has been proposed in our previous work [13].

The remainder of this work is organized as follows. In Section II, we describe the mobile channel model under con- 
sideration. In Section III, we explain the pilot-aided channel estimation including analytical expression of different interpolation techniques and transmission model. In Section IV, we present numerical results and we provide concluding remarks in Section V.

Notation: $\|.\| \|^{2}$ denotes Euclidean distance. (.) $)^{\prime}$ and (.) denote the first and second derivative of a given function, respectively. $\mathrm{E}[$.$] is statistical mean function.$

\section{Channel Model}

In this work, we utilize the mobile channel impulse response proposed in our previous study [14] as time-varying channel response. The channel impulse response is obtained through the non-sequential ray tracing feature of Zemax. As the first step, three dimensional simulation environment, a living room with a size of $6 \mathrm{~m} \times 6 \mathrm{~m} \times 3 \mathrm{~m}$ is created as illustrated in Fig. 1. Furthermore, several CAD models including human body, table, chairs, couch and coffee table are integrated into the living room. Moreover, 9 commercially available LEDs (Cree CR6-800L) with $40^{\circ}$ half view angle are located at the ceiling. The optical power for each luminary is 11 Watts. This yields an average illumination level of 153 lux which satisfies typical illumination requirements for home environment.

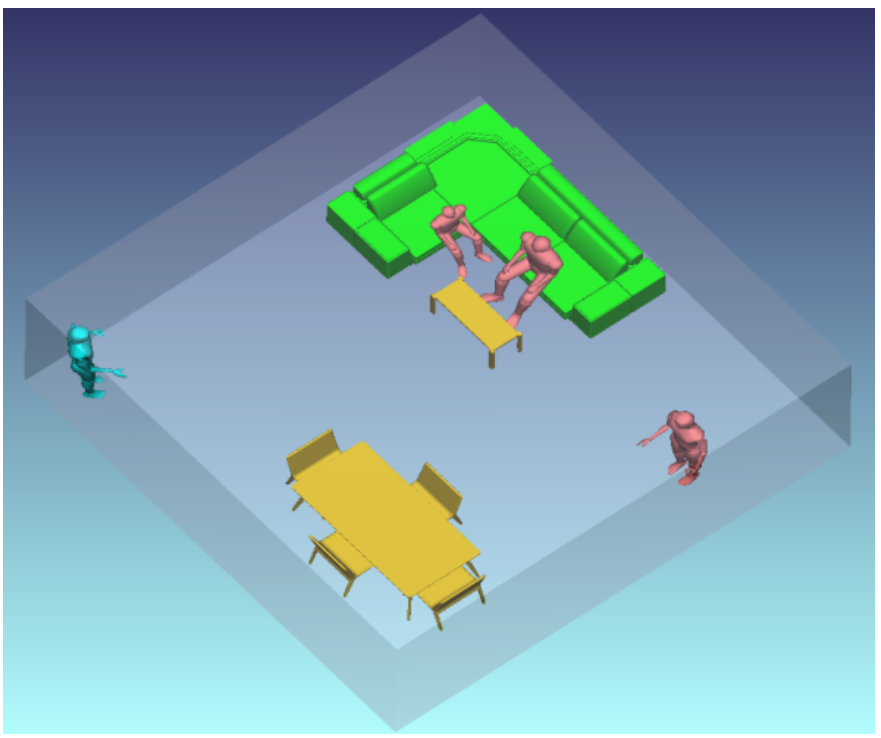

Figure 1: Living room under consideration.

We assume a human body holding PD included cell phone in hand next to ear, moves on a trajectory (see in Fig. 2) starting from the top-left corner within the room. The orientation of human body changes according to the direction of the way while the rotation and location of cell phone (i.e., $45^{\circ}$ rotation and at a height of $1.8 \mathrm{~m}$ ) in his/her hand are fixed with respect to his/her ear. The field-of-view (FOV) and the area of the detector are $85^{\circ}$ and $1 \mathrm{~cm}^{2}$ respectively. The ray tracing feature of Zemax is used in order to calculate the received optical power and path lengths from source to detector for each ray. These are used to obtain the channel impulse response (CIR) between the light sources and PD.

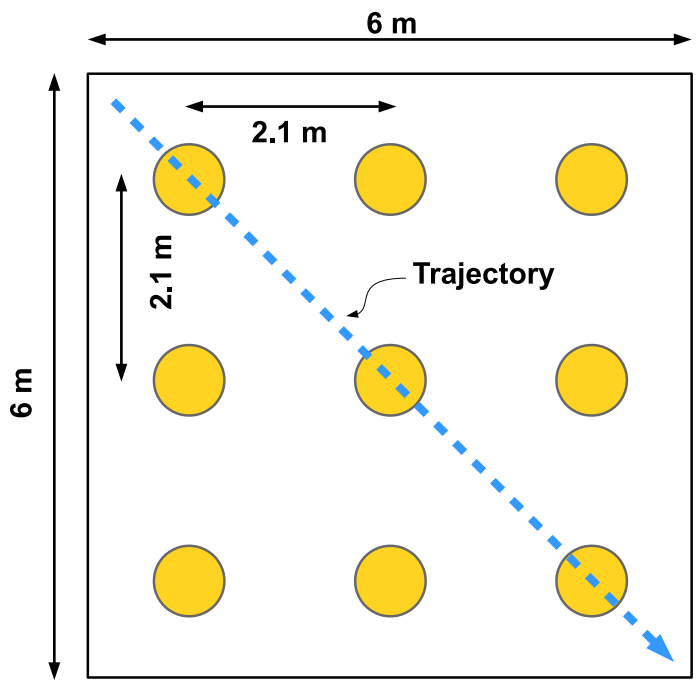

Figure 2: Movement trajectory.

We obtain CIRs for all points with $40 \mathrm{~cm}$ inter-distance along the trajectory. There are totally 19 sample points with respect to $40 \mathrm{~cm}$ inter distance. Then we interpolate them using spline interpolation technique whose expression is provided in the following section to approximate CIR as a continuous response. Let $h_{i, d}(t)$ denote the individual optical CIR between the $i^{\text {th }}$ luminary and UE at the distance of $d$ with respect to the reference point. The combined CIR at the UE can be obtained from

$$
h_{d}(t)=\sum_{i=1}^{N_{T}} h_{i, d}(t)
$$

where $N_{T}$ is the number of luminaries. In Fig. 3, we depicted the frequency-flat channel responses $\left(h_{d}=\int_{t=0}^{\infty} h(t) d t\right)$ with respect to different distances. Based on UE movements, we introduce mobile channel model: $h(t)=h_{d}$ considering that UE is far away from the start point with distance $d$ at the time of $t$.

\section{SySTEM MODEL}

Our system model is depicted in Fig. 4, we use single carrier (SC) $M$-pulse amplitude modulation (PAM) deployed transmission in indoor room environment described in previous section. Intensity levels of $M$ ordered SC-PAM are given by

$$
I_{m}=\frac{2 I}{M-1} m \text { for } m=0,1 \ldots M-1,
$$

where $I$ is the mean optical power emitted by LED. The bits are first mapped into PAM symbols denoted by $x[n]$ where $n \in\{0,1 \ldots P-2\}$ is the index of the symbols which are transmitted from LEDs to UE. One pilot symbol is inserted after $P-1$ PAM symbols. Here, $P$ denotes the pilot spacing, in other words, the period of pilots that are periodically inserted into symbol vector (s) for the purpose of downlink channel impulse response estimation (see Fig. 5). 


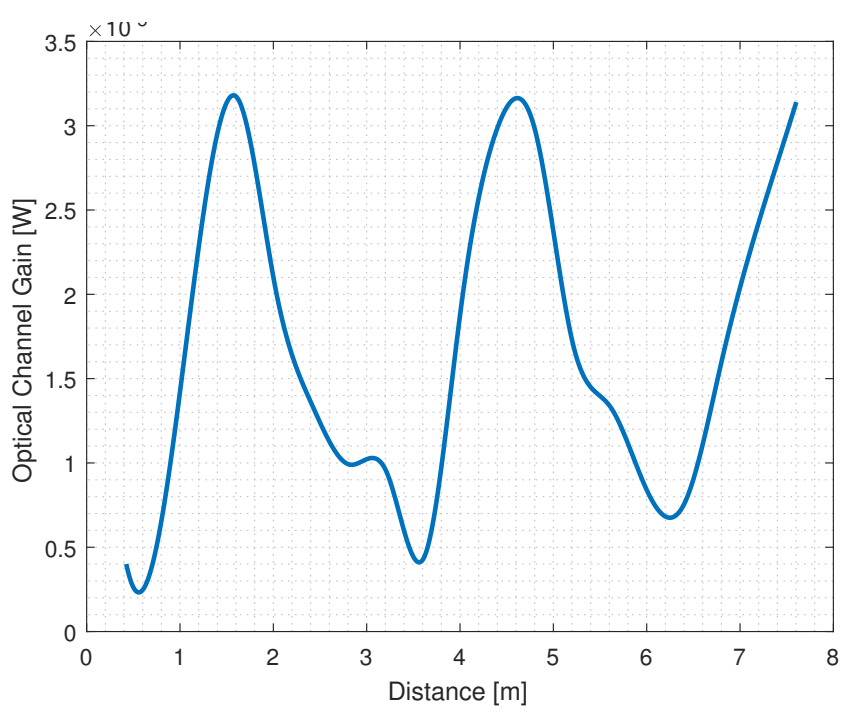

Figure 3: Optical channel gain vs. distance along trajectory under consideration.

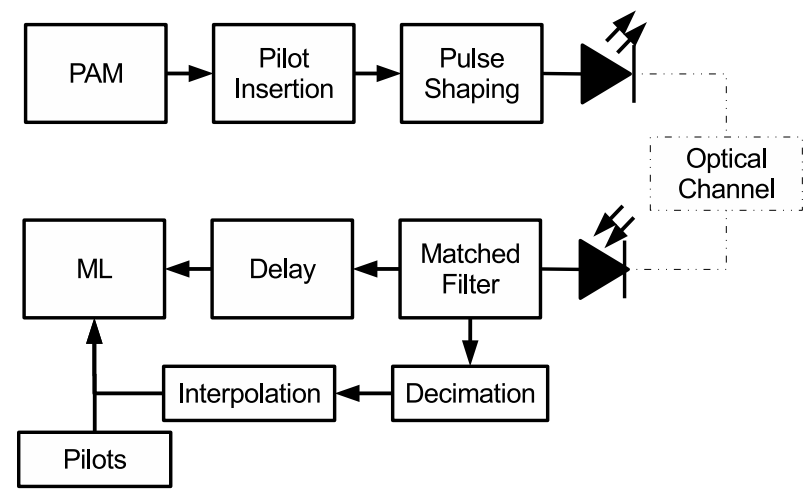

Figure 4: Pilot-aided transmission model.

The sequence is then passed through the pulse shaping filter and emitted by LEDs after digital-to-analog conversion process. The signal emitted by LEDs is denoted by $x(t)$. In this work, each LED transmits the same signal sequence and perfect synchronization between LEDs is assumed. The transmitted signal propagates through the optical channel and captured by PD available on UE. The received signal is obtained by

$$
y(t)=R x(t) h(t)+v(t),
$$

where $v(t)$ is additive white Gaussian noise (AWGN) term with power spectral density (PSD) of $N_{0}$ and $R$ is the responsivity of the PD. The received signal is passed through a matched filter and decimated with the rate of $1 / P$, starting from a point regarding to pilot symbol positions. The decimation outputs are interpolated and results are used as estimated channel response in Maximum Likelihood (ML) decision rule, which can be written as

$$
\hat{s}[n]=\underset{x \in I}{\operatorname{argmin}}\|y[n]-\hat{h}[n] R x\|^{2},
$$

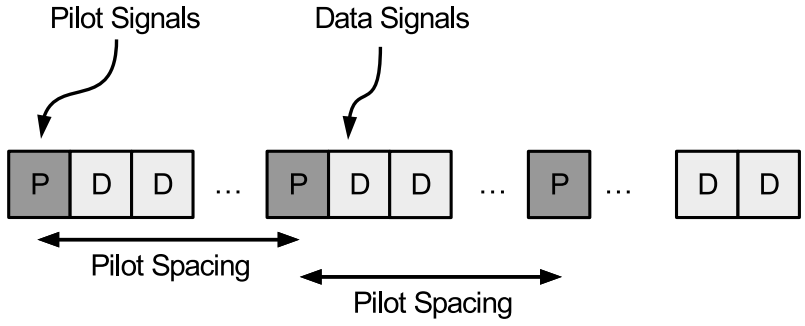

Figure 5: Transmitted frame structure

where I includes all possible symbols in $M$-PAM. At the interpolation step, different interpolation techniques (e.g., linear, nearest, spline, pchip etc.) can be employed. They have different complexities and yield different performance results.

\section{A. Interpolation Techniques}

For a point denoted by $x$ within a particular interval between $x_{k}$ and $x_{k+1}$ with a discrete function of $f\left(x_{k}\right)$ given a pair of $\left(x_{k}, f\left(x_{k}\right)\right)$, linear interpolation formula can be written as

$$
f(x)=f\left(x_{k}\right)+\left(x-x_{k}\right) \frac{f\left(x_{k+1}\right)-f\left(x_{k}\right)}{x_{k+1}-x_{k}} .
$$

Then, a straight line is fitted through the points of $\left(x_{k}, f\left(x_{k}\right)\right)$ and $\left(x_{k+1}, f\left(x_{k+1}\right)\right)$. The second interpolation method, nearest interpolator, fills the points, $x$, within the range of $x_{k}$ and $x_{k+1}$, with either $f\left(x_{k}\right)$ or $f\left(x_{k+1}\right)$ regarding to which one of the $x_{k}$ and $x_{k+1}$ is more close to $x$. Third method, spline interpolation technique, includes second derivative of $f\left(x_{k}\right)$ and $f\left(x_{k+1}\right)$. The analytical formula can be written as

$$
\begin{aligned}
& f(x) \quad=\frac{x_{k+1}-x}{x_{k+1}-x_{k}} f\left(x_{k}\right)+\frac{x-x_{k}}{x_{k+1}-x_{k}} f\left(x_{k+1}\right)+ \\
& \frac{\left(\left(\frac{x_{k+1}-x}{x_{k+1}-x_{k}}\right)^{3}-\left(\frac{x_{k+1}-x}{x_{k+1}-x_{k}}\right)\right)\left(x_{k+1}-x_{k}\right)^{2}}{6} f\left(x_{k}\right)^{\prime \prime}+ \\
& \frac{\left(\left(\frac{x-x_{k}}{x_{k+1}-x_{k}}\right)^{3}-\left(\frac{x-x_{k}}{x_{k+1}-x_{k}}\right)\right)\left(x_{k+1}-x_{k}\right)^{2}}{6} f\left(x_{k+1}\right)^{\prime \prime} \text {. }
\end{aligned}
$$

Spline interpolator aims to get continuity in both first and second derivatives within the intervals and produces smoother results. Its accuracy increases for smooth function obtained interpolated data. On the other hand, accuracy of the last method, pchip, whose formula can be written as

$$
\begin{gathered}
f(x)=\frac{3\left(x_{k+1}-x_{k}\right)\left(x-x_{k}\right)^{2}-2\left(x-x_{k}\right)^{3}}{\left(x_{k+1}-x_{k}\right)^{3}} f\left(x_{k+1}\right)+ \\
\frac{\left(x_{k+1}-x_{k}\right)^{3}-3\left(x_{k+1}-x_{k}\right)\left(x-x_{k}\right)^{2}+2\left(x-x_{k}\right)^{3}}{\left(x_{k+1}-x_{k}\right)^{3}} f\left(x_{k}\right)+ \\
\frac{\left(x-x_{k}\right)^{2}\left(\left(x-x_{k}\right)-\left(x_{k+1}-x_{k}\right)\right)}{\left(x_{k+1}-x_{k}\right)^{2}} f\left(x_{k+1}\right)^{\prime}+ \\
\frac{\left(x-x_{k}\right)\left(\left(x-x_{k}\right)-\left(x_{k+1}-x_{k}\right)\right)^{2}}{\left(x_{k+1}-x_{k}\right)^{2}} f\left(x_{k}\right)^{\prime},
\end{gathered}
$$

yields high accuracy when data is not an output of smooth function with the same computational effort.

\section{NumericAl Results}

In this section, we investigate the SER and MAE performance with respect to different $M$ ordered PAM, different interpolation techniques, various pilot signal periods, and 
numerous UE speeds in indoor room environment through Monte-Carlo simulations. MAE is calculated from the following equation, MAE $=\mathrm{E}\left[\frac{h[n]-\hat{h}[n]}{h[n]}\right]$. During simulations, sampling interval $\left(T_{S}\right)$, responsivity $(R)$, and noise PSD $\left(N_{0}\right)$ are set to $1 \mathrm{usec}, 0.28 \mathrm{~A} / \mathrm{W}$ [15], and $10^{-22} \mathrm{~W} / \mathrm{Hz}$ [15], respectively.

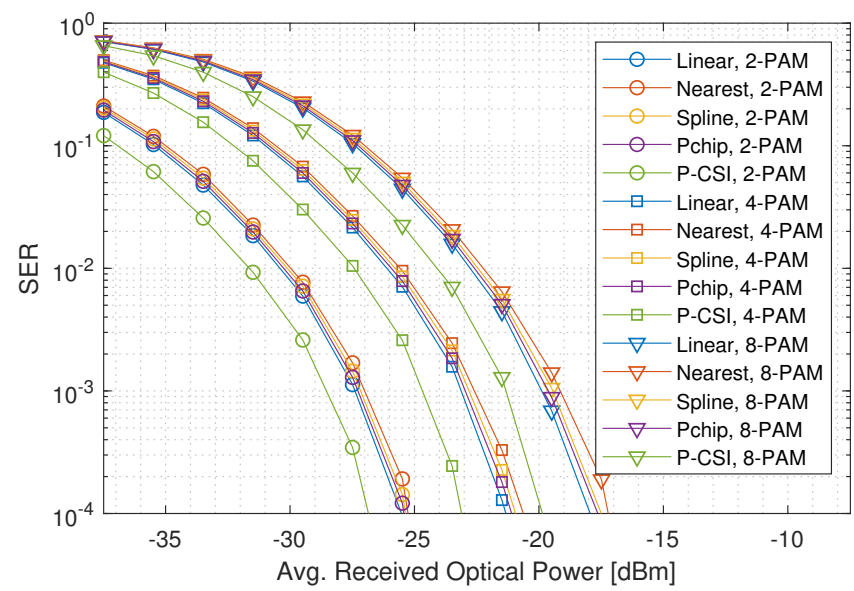

(a)

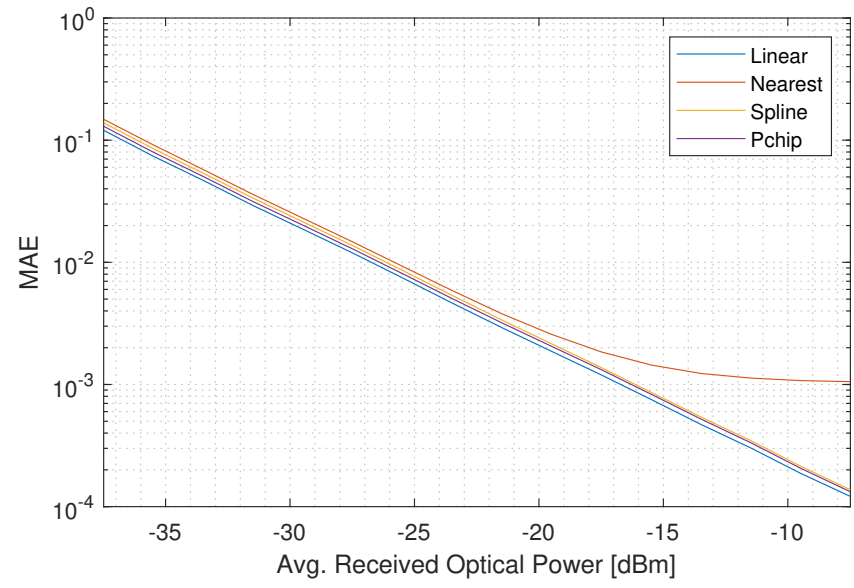

(b)

Figure 6: SER and MAE performance of different interpolation techniques using different modulation with $1 \mathrm{~m} / \mathrm{s}$ UE speed and $1 / 10000$ pilot signal period.

In Fig. 6, we present SER and MAE performance with respect to different modulation orders and interpolation methods for $1 / 10000$ pilot signal period and $1 \mathrm{~m} / \mathrm{s}$ UE speed in indoor room environment. We further depict perfect channel state information (CSI) availability at the UE case as benchmark to evaluate the performance of interpolation techniques. The results reveal that linear interpolation technique gives the best performance among the explored techniques. It outperforms pchip interpolation with the gain of $0.1 \mathrm{~dB}$ for 2-PAM at the SER target of $10^{-3}$. The gain of linear method over spline and nearest techniques is $0.25 \mathrm{~dB}$ and $0.7 \mathrm{~dB}$, respectively, for the same modulation order and at the same SER target. The performance degradation compared to perfect CSI is $1.2 \mathrm{~dB}$. On the other hand, when we increase the modulation orders

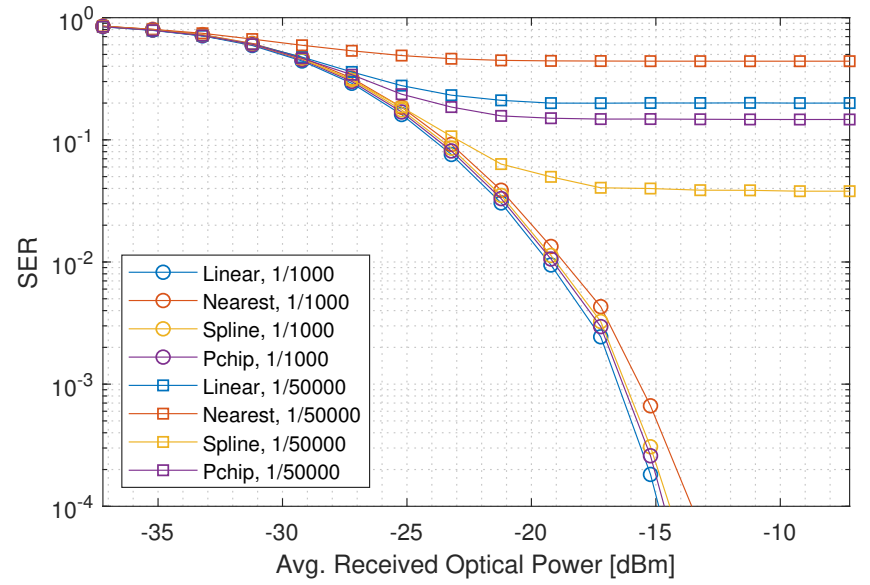

(a)

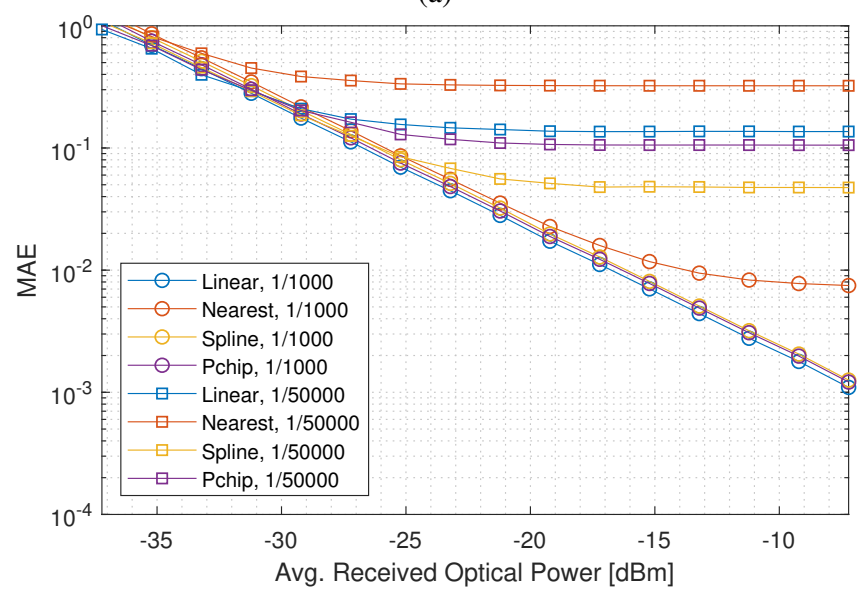

(b)

Figure 7: SER and MAE performance of different interpolation techniques using different pilot signal periods with $7 \mathrm{~m} / \mathrm{s}$ UE speed and 16-PAM deployment.

such as $4-\mathrm{PAM}$, and $8-\mathrm{PAM}$, the degradation with respect to P-CSI is increased by 0.2 and $0.4 \mathrm{~dB}$ respectively. In Fig. 6.b, MAE results are depicted. It can be observed that the average received optical power of $-16.8 \mathrm{dBm}-16.4 \mathrm{dBm},-16.2$ $\mathrm{dBm}$, more than $-10 \mathrm{dBm}$ is required in order to achieve $10^{-3}$ MAE for linear, pchip, spline, and nearest interpolation techniques, respectively. The lowest estimation error is obtained with the linear interpolation technique. Hence, it requires the lowest optical power in order to achieve target SER value.

In Fig. 7, we present SER and MAE performance with respect to different pilot signal periods and interpolation methods under consideration of $7 \mathrm{~m} / \mathrm{s}$ UE speed in indoor room environment and 16-PAM deployed transmission. Pilot periods of 1 per 1000 (denoted by $1 / 1000$ ) and 1 per 50000 (denoted by $1 / 50000$ ) symbols are considered. The results reveal that increment on pilot signal period results in degradation on SER and MAE performance for all considered interpolation techniques. The nearest interpolation technique is the weakest one against increasing pilot periods. It is followed by linear and pchip techniques, respectively. Spline is the most 


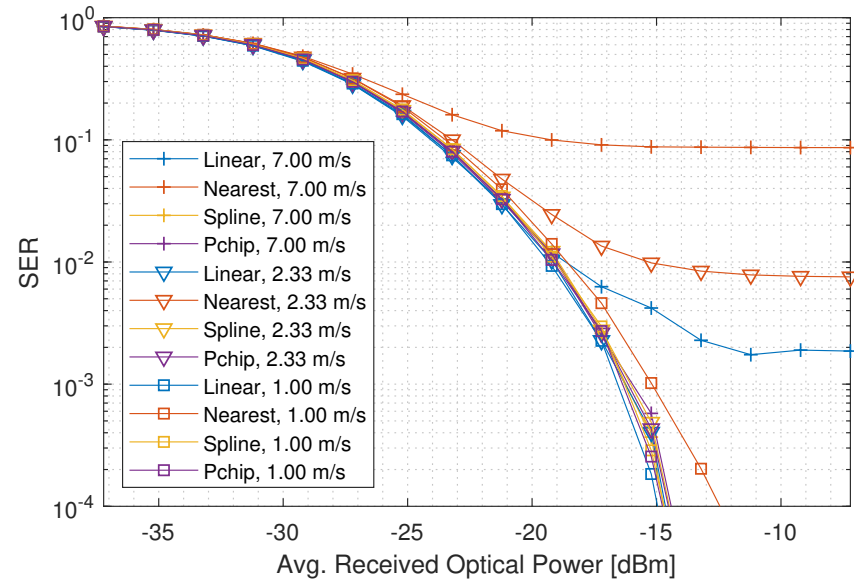

(a)

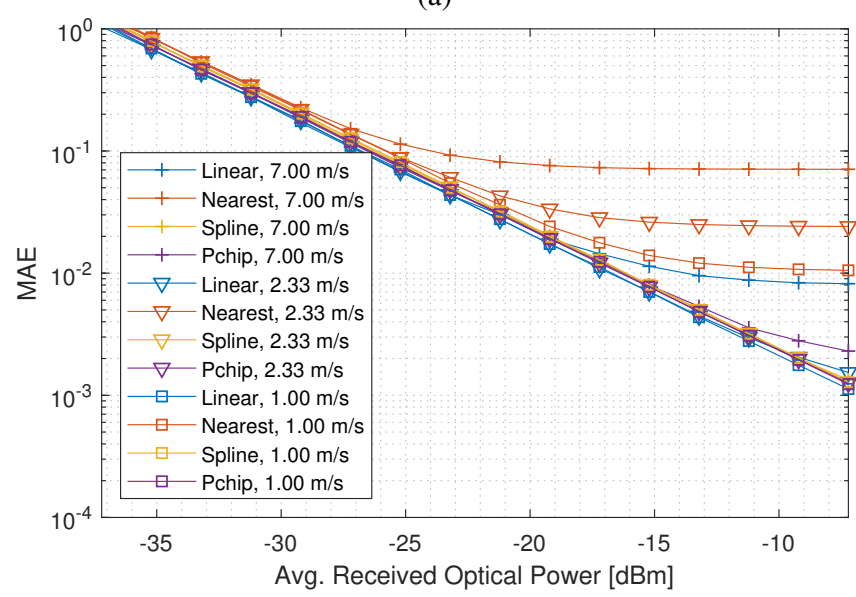

(b)

Figure 8: SER and MAE performance of different interpolation techniques with different UE speed values, 16-PAM deployment, and 1/10000 pilot signal period.

robust one even though it gives worse performance than linear interpolator in low pilot periods.

In Fig. 8, we present SER and MAE performance with respect to different UE speeds and interpolation methods under consideration of 16-PAM deployed transmission and pilot period of 1 per 10000 symbols. It can be observed that similar to previous case, increment on UE speeds results in degradation on SER performance for any interpolation technique under consideration. The nearest interpolation technique is the weakest one against high UE speeds as a consequence of fast varying channel response at the same pilot signal and same estimation period. It is followed by linear and pchip techniques, respectively. Spline is the most robust one even though it performs worse than linear interpolator at low UE speeds.

\section{CONCLUSION}

In this paper, we investigate the SER and MAE performance of linear, nearest, spline and pchip interpolation techniques over realistic time-varying channel conditions. The results are compared with each other considering different pilot signal transmission rate, modulation order, and speed of UE moving in indoor room environment. The results reveal that low-complexity linear interpolation technique can be used for slowly-varying channel conditions with low pilot signal periods. On the other hand, for higher UE speeds leading fast varying channel response and considering the increased pilot signal periods, spline interpolator or pchip can be selected to avoid from performance degradation on SER and provide a reliable transmission.

\section{REFERENCES}

[1] D. Karunatilaka, F. Zafar, V. Kalavally, and R. Parthiban, "LED Based Indoor Visible Light Communications: State of the Art," IEEE Communications Surveys Tutorials, vol. 17, pp. 1649-1678, thirdquarter 2015.

[2] M. Uysal, F. Miramirkhani, O. Narmanlioglu, T. Baykas, and E. Panayirci, "IEEE 802.15.7r1 Reference Channel Models for Visible Light Communications," IEEE Communications Magazine, vol. 55, pp. 212-217, January 2017.

[3] T. Komine, J. H. Lee, S. Haruyama, and M. Nakagawa, "Adaptive equalization system for visible light wireless communication utilizing multiple white LED lighting equipment," IEEE Transactions on Wireless Communications, vol. 8, pp. 2892-2900, June 2009.

[4] O. Narmanlioglu, R. C. Kizilirmak, and M. Uysal, "Relay-assisted OFDM-based visible light communications over multipath channels," in 2015 17th International Conference on Transparent Optical Networks (ICTON), pp. 1-4, July 2015.

[5] Y. S. Hussein, M. Y. Alias, and A. A. Abdulkafi, "On performance analysis of $1 \mathrm{~s}$ and mmse for channel estimation in vlc systems," in 2016 IEEE 12th International Colloquium on Signal Processing Its Applications (CSPA), pp. 204-209, March 2016.

[6] O. ayli, H. Doan, and E. Panayirci, "On channel estimation in dc biased optical ofdm systems over vlc channels," in 2016 International Conference on Advanced Technologies for Communications (ATC), pp. 147151 , Oct 2016.

[7] X. Yang, Z. Min, T. Xiongyan, W. Jian, and H. Dahai, "A post-processing channel estimation method for dco-ofdm visible light communication," in 2012 8th International Symposium on Communication Systems, Networks Digital Signal Processing (CSNDSP), pp. 1-4, July 2012.

[8] T. Zhang, S. Guo, H. Chen, F. Zhong, and C. Ma, "Enhancing the bit error rate of indoor visible light communication systems using adaptive channel estimation algorithm," IET Communications, vol. 9, no. 4, pp. 501-507, 2015.

[9] H. Dogan, O. ayl, and E. Panayirci, "Pilot assisted channel estimation for asymmetrically clipped optical ofdm over visible light channels," in 2016 IEEE International Black Sea Conference on Communications and Networking (BlackSeaCom), pp. 1-4, June 2016.

[10] C. Chen, W. D. Zhong, and L. Zhao, "Sparse bayesian rvm regression based channel estimation for im/dd ofdm-vlc systems with reduced training overhead," in 2017 IEEE International Conference on Communications Workshops (ICC Workshops), pp. 162-167, May 2017.

[11] X. Chen and M. Jiang, "Adaptive statistical bayesian mmse channel estimation for visible light communication," IEEE Transactions on Signal Processing, vol. 65, pp. 1287-1299, March 2017.

[12] X. Chen and M. Jiang, "Enhanced bayesian mmse channel estimation for visible light communication," in 2016 IEEE 27th Annual International Symposium on Personal, Indoor, and Mobile Radio Communications (PIMRC), pp. 1-6, Sept 2016.

[13] F. Miramirkhani, O. Narmanlioglu, M. Uysal, and E. Panayirci, "A Mobile Channel Model for VLC and Application to Adaptive System Design," IEEE Communications Letters, vol. 21, pp. 1035-1038, May 2017.

[14] F. Miramirkhani and M. Uysal, "Channel Modeling and Characterization for Visible Light Communications," IEEE Photonics Journal, vol. 7, pp. 1-16, Dec 2015.

[15] J. Grubor and S. Randel and K. D. Langer and J. W. Walewski, "Broadband Information Broadcasting Using LED-Based Interior Lighting,' Journal of Lightwave Technology, vol. 26, pp. 3883-3892, Dec 2008. 